\title{
Can Dietary Instructions Delivered Through Mobile Application Reduce Sweet Score among Adolescents in Chennai, India?-A Randomized Controlled Preventive Trial
}

\author{
Shyam Sivasamy ${ }^{1}$, Arun Kumar Sundaragopal ${ }^{2}$, Preetha E. Chaly ${ }^{3}$, \\ Nijesh J.E. ${ }^{4}$, Vaishnavi Selvaperumal ${ }^{5}$, Anusha Divvi ${ }^{6}$ \\ ${ }^{1,3,4}$ Dept of Public Health Dentistry, Faculty of Dentistry, Meenakshi Academy of Higher Education and \\ Research, Chennai, India. ${ }^{2}$ Dept of Public Health Dentistry, Sri Venkateswara Dental College and Hospital, \\ Chennai, India. ${ }^{5}$ Dept of Public Health Dentistry, Tagore Dental College and Hospital, Chennai, India. ${ }^{6}$ Dept of \\ Public Health Dentistry, Indira Gandhi Institute of Dental Sciences, Sri Balaji Vidhyapeeth, Pondicherry, India.
}

\begin{abstract}
Background: Mobile health (mhealth) intervention has been proved to be effective in weight loss, diabetes control and tobacco cessation Objective: To assess the effectiveness of mhealth intervention to reduce consumption of cariogenic diet. Design and setting: Randomized controlled trial in field setting Materials and method: Forty two adolescents who were in the "Watch-out" zone of sweet score were recruited for the study. The subjects were randomly allotted into the test group $(n=21)$ and the control group $(n=21)$. A three day diet diary was recorded from all study subjects and the average sweet score was assessed at baseline and at post intervention. The subjects in the control group received standard one to one dietary instructions tailored to the needs of the subjects only once at the start of the study. Subjects in the test group received tailored dietary instructions once daily through mobile application (Whatsapp). Intra-group comparison was made using Wilcoxon signed rank test and inter-group comparison was made using Mann Whitney U test. $\mathrm{P}$ value $<0.05$ was considered statistically significant. Results: The sweet score for the control group at baseline was $20.9 \pm 6.06$ while the post test score was $13.16 \pm 4.37$ and the difference was statistically significant $(\mathrm{p}=0.002)$. The sweet score for the test group at baseline was $18.02 \pm 4.03$ while the post test score was $14.5 \pm 7.74$ and the difference was statistically significant $(\mathrm{p}=0.03)$. However, there was no statistically significant difference in sweet score between the two groups at the end of the trail. Conclusion: mhealth intervention is effective in reducing sweet score among adolescents.
\end{abstract}

Keywords: Mobile application, dietary instructions, cariogenic diet, sweet score.

\section{Introduction}

Non communicable diseases (NCDs) are the rising public health problem in the developing countries contributing to high morbidity and mortality rate. It is collectively responsible for almost $70 \%$ of all deaths worldwide. Low- and middle-income countries account for $3 / 4^{\text {th }}$ of deaths due to NCD which includes nearly

\section{Corresponding Author:}

\section{Arun Kumar Sundaragopal}

Dept. of Public Health Dentistry, Sri Venkateswara

Dental College and Hospital, Chennai, India

e-mail: ak.s29@live.com
$80 \%$ of all premature deaths $\left[^{1}\right]$. Four major risk factors namely tobacco use, physical inactivity, the harmful use of alcohol and unhealthy diet have been attributed for the rise in NCDs across the world. . Increased consumption of food containing highly refined sugar is an important behavioral change which is implicated as a common risk factor for many NCDs like obesity, diabetes and dental caries. Hence, interventions targeting unhealthy diet containing high levels of refined sugar can help prevent these diseases/conditions.

Dental caries is the most common chronic condition of both childhood and adulthood affecting more than 2.4 billion adults and 621 million children worldwide $\left.{ }^{2}\right]$. It is the $10^{\text {th }}$ most common disease affecting children. 
Untreated dental caries may cause sensitivity, pain, absenteeism from school and workplace, ultimately affecting the oral health related quality of life. Among 291 diseases investigated for causing disability, untreated dental caries was placed at $80^{\text {th }}$ position $\left[{ }^{3}\right]$.

Initiation and progression of dental caries can be prevented by limiting the exposure to cariogenic diet. Diet counselling and dietary instructions are hence advocated as a mainstay in the primary prevention of dental caries. Conventionally, diet instructions in diet counselling is delivered by trained personnel on a one on one basis through which consensus is reached between the counsellor and the subject on required dietary modifications to control dental caries. Despite being an effective tool for caries prevention, it is quite challenging to bring about dietary modification as it requires high level of motivation, and periodic reinforcement $\left[{ }^{4}\right]$. Hence, newer method of delivering dietary instruction which reinforce and motivate subjects on a regular basis might have higher success rate in bringing about the desired change in diet. One such recent technology to deliver health information is through mobile applications using smartphones, popularly referred to as mobile health interventions (mHealth intervention) $\left.{ }^{5}\right]$.

Mobile health interventions have been proved to be effective in various health promotion programs involving, tobacco cessation, weight reduction and diabetes control[ $\left.{ }^{6-8}\right]$. Although many smartphone apps related to diet and nutrition are available, relatively few have been tested for their effectiveness in promoting health. There is an actual paucity in literature regarding the effectiveness of mobile technology based dietary instruction to reduce the intake of diet rich in highly refined sugar. Hence, a randomized controlled trial was conducted to evaluate the effectiveness of mobile technology based dietary instructions and to compare its effectiveness with standard one to one dietary instructions on reducing cariogenic diet rich in highly refined sugar among healthy adolescents in Chennai. The research hypothesis was that dietary instructions delivered through mobile application are as effective as dietary instruction by standard one to one method.

\section{Materials and Method}

A double blinded randomized controlled field trial employing concurrent parallel design was conducted among 18 years old apparently healthy college going adolescents. This trial conforms to the recommendations of CONSORT statement $2010\left[^{9}\right]$. Prior to the start of the study, a detailed protocol was prepared which was scrutinized and ethical clearance was obtained from the Institutional Review Board bearing the reference number MADC/IRB-XIII/2017/287. No changes were made in the methodology after obtaining the ethical clearance.

A multiphase random sampling technique was employed to recruit the study subjects from a randomly selected Arts and Science College in Chennai city. In stage I, all students in the college $(n=946)$ were screened to identify subjects who have three or more carious lesions in their oral cavity using mouth mirror and dental explorer based on WHO criteria 2013 [10]. A total of 124 subjects had more than three active carious lesions. In phase II, the identified 124 subjects were asked to submit their 24 hour diet chart to assess their Sweet score based on Nizel and Papas criteria $\left[{ }^{11}\right]$. Subjects whose sweet score was $\geq 15$ (Watch out zone) were identified. In stage III, 61 subjects in the Watch-out zone of sweet score were identified and were considered eligible for the dietary intervention. The study subjects were recruited between August and October 2018. The subjects were explained about the purpose of the study and informed voluntary written consent was obtained prior to the start of the study. Subjects who were active in using Whatsapp mobile application were eligible for the study. Subjects who have special dietary requirements were excluded from the study.

The sample size for the study was calculated by fixing alpha error at $5 \%$ and beta error at $20 \%$. Expecting a difference of five units in the sweet score between the two groups, the sample size was estimated to be 15 subjects in each group. Expecting a drop rate of 30\%, the sample size was increased to 21 subjects in each group. Forty two subjects were randomly selected from the list of 61 eligible subjects by simple random sampling using lottery method for the trial and randomized into the test group and the control group. A random allocation sequence was generated using the online randomization software (www.randomizer.org) using 1:1 ratio. The sequence was generated by an independent personnel not involved in the study (NJE). Based on the sequence, opaque envelops were prepared, serially numbered and used for the allotting the subjects to the test group and the control group with 21 subjectsin each group. The investigator who assessed the diet diary and the statistician who analysed the data were blinded to the intervention. 
Baseline data on sweet score of the subjects was assessed by recording a diet chart. The subjects were given a three day diet diary and were instructed to enter details of all food items consumed, its quantity in household measurement, details regarding sugar added, and timing of intake. The primary outcome measure was the average sweet score which was calculated for each individual by summing up the sweet score for each day and dividing it by three. The sweet score for each food item containing sugar was allotted based on the Nizel and Papas criteria $\left[{ }^{11}\right]$. The diet diaries collected from the study subjects were coded to ensure blinding and was assessed by two authors (AKS and SS). The dietary instructions for each subject were tailored according to their needs based on the recommendations given by Nizel and Papas.

Subjects in the test group were sent dietary instructions which were tailored to their needs, on a daily basis through mobile application (Whatsapp). They were also sent motivational picture messages and were encouraged to clear their doubts about the dietary changes through messaging using the same application. Daily reminders like "Are you following the dietary instructions?" were also sent to the subjects in the test group. Subjects in the control group received standard one to one dietary instructions once at the beginning of the trial. The data as well as the one on one diet instructions for the control group were conducted at the college premises during the working hours. The subjects were given choices to have hot or cold beverages without sugar or if they found it difficult to have beverages without adding sugar they were asked to reduce the frequency of consuming the beverage or at least try to taper the number of times of beverage consumption. They were also instructed to avoid candies, carbonated drinks, ice-creams and biscuits and include snacks like popcorn, peanuts and sandwiches in their diet. At the end of 4 weeks, subjects in the test group and control group were asked to record a three day diet diary, using which the post intervention sweet score was calculated.

Data analysis was done per protocol. The data was entered in Microsoft Excel and analysed using SPSS version 19. Descriptive tables were made expressing quantitative variables in terms of mean and standard deviation. Normality of the data was assessed using Shapiro-Wilk test and was found to be nonnormally distributed. Differences that existed between the intervention and control groups were tested for statistical significance using Mann-Whitney $U$ test. Wilcoxon sign-rank test was used to compare difference between baseline and post-intervention sweet score in both control as well as the test group. P value $<0.05$ was considered to be statistically significant.

Table 1: Inter and intra group comparison of sweet scores at the beginning of the study and at the end of 4 weeks

\begin{tabular}{|l|c|c|c|}
\hline Groups & Baseline sweet score & Post intervention sweet score & p value* $^{*}$ \\
\hline Control & $20.9 \pm 6.06$ & $13.16 \pm 4.37$ & 0.002 \\
\hline Test & $18.02 \pm 4.03$ & $14.5 \pm 7.74$ & 0.03 \\
\hline p value $^{\dagger}$ & 0.08 & 0.77 & \\
\hline
\end{tabular}

* Wilcoxon signed rank test, $\uparrow$ Mann Whitney U test

\section{Results}

A total of 42 subjects were randomly divided into test group and control group with 21 subjects in each group. Among the 21 subjects who received the assigned intervention in each group,totally 12 subjects were eliminated from data analysis either due to loss to follow up or due to incomplete data as shown in Figure 1. Thus the data obtained from 15 subjects were available in each for final data analysis. The mean age of the study subjects was $18 \pm 0.49$ years and $80 \%$ $(n=24)$ were females. The mean number of caries teeth of the study subjects was $3.83 \pm 0.28$ and there was no significant difference between the test group and the control group. The mean sweet score in the control group was $20.9 \pm 6.06$ at the baseline which reduced to $13.16 \pm 4.37$ at the end of four weeks and this difference was statistically significant $(\mathrm{p}=0.002)$. In the test group the mean sweet score was $18.02 \pm 4.03$ at baseline which reduced to $14.5 \pm 7.74$ and this difference was statistically significant $(\mathrm{p}=0.03)$. Table 2 shows that the difference in 
the mean percentage reduction in sweet score between the test group and the control group was not statistically significant. Among the 15 subjects in the control group who were in the Watch out zone at baseline, two subjects moved to the Excellentzone and three subjects moved to the Good zone of sweet score as per Nizel and Papas criteria. Similarly, among 15 subjects in the test group, four subjects moved to the Excellent zone and one subject moved to the Good zone of sweet score as per Nizel and Papas criteria.

Table 2: Intergroup comparison of mean percentage reduction in sweet score before and after intervention

\begin{tabular}{|l|l|c|}
\hline Groups & $\begin{array}{l}\text { Mean percentage reduction in } \\
\text { sweet score }\end{array}$ & p value* \\
\hline Control & $34.75 \pm 26.58$ & 0.16 \\
\hline Test & $22.35 \pm 31.00$ & \\
\hline
\end{tabular}

*Mann Whitney U test

\section{Discussion}

The aim of the present study was to assess the effectiveness of mobile technology using Whatsapp to reduce the sweet score among adolescents. The results of this randomized controlled preventive trial showed that daily reminders sent to subjects' mobile phone through Whatsapp mobile application was effective in reducing the Sweet score based on Nizel and Pappas criteria. mHealth interventions are consumer centered and assist the health care providers in delivering health education as well as patient management $\left[{ }^{12,13}\right]$. India,being the second highest mobile phone users in the world, the study resultareof practical importance $\left[{ }^{14}\right]$.

Adolescents were included in this trial as they have the ability to make informed decision and can process health information in an effective manner. By the time an individual reaches adolescence, he/she can exercise his preference over the diet and can opt for healthy dietary practices. Further in this study, adolescents were selected as they have access to smart phones and can comprehend the health information sent through Whatsapp.

Past dental caries is considered to be the best predictor of future dental caries $\left[{ }^{15,16}\right]$. Hence, subjects with at least three carious lesions were recruited in the present study as they can be considered high risk population for future dental caries and hence require diet counseling. Further, the subjects' need for diet counseling was verified by collecting a one day diet chart. Only those who were in the Watch out zone of sweet score were recruited for the study. Multiphase random sampling method was employed in the study to facilitate the identification of eligible subjects in the study.

Whatsapp was preferred over short messaging services since the former had the facility to know whether the daily reminders have been sent and subsequently seen by the recipient. In Whatsapp mobile application, if a message has been sent successfully a single grey tick appears besides the message, and it turns to double grey ticks if the message has been successfully delivered to the recipient. The double grey ticks later turns blue once the recipient has seen the message. This in built check mechanism in Whatsapp helped the investigators to follow if the subjects read the daily reminders. Whatsapp was preferred over other messaging applications because it was a freely downloadable application which was highly popular among adolescents.

However, the results should be interpreted by keeping the limitations of the study in mind. Though the study was conducted for a short duration of four weeks, literature shows that many dietary intervention trials were conducted for such a duration. Although the study saw a high loss to follow up, we believe that the power of the study would not be affected as such a situation was anticipated and the sample size was adjusted accordingly at the start of the study. For the same reason, drop out analysis was also not carried out. There is also a possibility of hawthorne effect as well as social desirability bias might have crept into the study. Both the method of diet instructionnamely the standard one on one counseling as well as the dietary instructions delivered through mobile application were effective in bringing about a significant reduction in the sweet score. This study was the first attempt to use mobile application to deliver dietary instructions to reduce the sweet score and hence direct comparison of the study results with previous literature could not be performed. The results of this study can be as the study followed rigorous methodology to select the study subjects in a random manner and the size of the sample was adequate despite the drop out Dietary instructions delivered through mobile application can be used in those instances where there are constraints of time and access to health professionals. This method can be considered cost effective method for delivering health information to a wide population given the high usage of smart phones among adolescents. The present study assessed the 
short term effectiveness of the intervention in reducing the sweet score of adolescents. However, the long term impact of mobile application based intervention needs to be assessed in future.

\section{Conclusion}

It can be concluded that dietary instructions delivered through mobile application is effective in reducing the sweet score.

\section{Ethical Clearance: Nil}

Source of Funding: Meenakshi Academy of Higher Education and Research, Chennai, India

\section{Conflict of Interest: Nil}

\section{References}

1. https://www.who.int/ncds/introduction/en/ accessed on 17.3.2019

2. Kassebaum NJ, Bernabé E, Dahiya M, Bhandari B, Murray CJ, Marcenes W. Global burden of untreated caries: a systematic review and metaregression. Journal of dental research. 2015 May;94(5):650-8.

3. Peres KG, Nascimento GG, Peres MA, Mittinty MN, Demarco FF, Santos IS, Matijasevich A, Barros AJ. Impact of prolonged breastfeeding on dental caries: a population-based birth cohort study. Pediatrics. 2017 Jul 1;140(1).

4. Moynihan P, Petersen PE. Diet, nutrition and the prevention of dental diseases. Public health nutrition. $2004 \mathrm{Feb}$;(1a):201-26.

5. Free C, Phillips G, Felix L, Galli L, Patel V, Edwards P. The effectiveness of M-health technologies for improving health and health services: a systematic review protocol. BMC research notes. 2010 Dec 1;3(1):250.

6. Ghorai K, Akter S, Khatun F, Ray P. mHealth for smoking cessation programs: a systematic review. Journal of personalized medicine. 2014 Sep;4(3):412-23.
7. Martin CK, Gilmore LA, Apolzan JW, Myers CA, Thomas DM, Redman LM. Smartloss: a personalized mobile health intervention for weight management and health promotion. JMIR mHealth and uHealth. 2016;4(1):e18.

8. Muralidharan S, Ranjani H, Anjana RM, Allender S, Mohan V. Mobile health technology in the prevention and management of type 2 diabetes. Indian journal of endocrinology and metabolism. 2017 Mar;21(2):334.

9. Schulz KF, Altman DG, Moher D, Consort Group. CONSORT 2010 statement: updated guidelines for reporting parallel group randomised trials. Trials. 2010 Dec 1;11(1):32.

10. Petersen PE, Baez RJ, World Health Organization. Oral health surveys: basic method.

11. Abraham EN. Athens S. Papas Nutrition in clinical dentistry.

12. Akter S, Ray P. mHealth-an ultimate platform to serve the unserved. Yearbook of medical informatics. 2010;19(01):94-100.

13. Gagnon MP, Ngangue $\mathrm{P}$, Payne-Gagnon J, Desmartis M. m-Health adoption by healthcare professionals: a systematic review. Journal of the American Medical Informatics Association. 2016 Jan 1;23(1):212-20.

14. Elangovan, R. and Arulchelvan, S., 2013. A study on the role of mobile phone communication in tuberculosis DOTS treatment. Indian journal of community medicine: official publication of Indian Association of Preventive \& Social Medicine, 38(4), p.229.

15. Vargas CM, Ronzio CR. Disparities in early childhood caries. InBMC oral health 2006 Jun 1 (Vol. 6, No. S1, p. S3). BioMed Central.

16. Carounanidy U, Sathyanarayanan R. Dental caries: A complete changeover (Part II)-Changeover in the diagnosis and prognosis. Journal of conservative dentistry: JCD. 2009 Jul;12(3):87. 